\title{
DESCARTES, LE "BON SENS", LA LOGIQUE ET LES VÉRITÉS ÉTERNELLES
}

Jacques Bouveresse

\section{Descartes, Leibniz et la démonstration}

Dans un article publie en 1986, lan IJacking a opposé de la façon suivante l'attitude de Descartes et celle de Leibniz à l'égard de la démonstration:

\begin{abstract}
Leibniz savait ce gu'est une démonstmation; Descartes ne le savait pas. Si l'on préle à ce lảit l'attention qu'il exige, cela aidè à résotddre certains problèmes d'interprétation qui ont tendancé à deverenir insaisissables. Ce n'est pas mon but principal aujourd' luti. Je m'intéresse davantage à la prélistoire qu'à l'histoire. Le concept leibnizien de la démonstration est presque le mêtne que le nỏtre. II n'a pas existé jusqu'à une époque qui est en gros la sienne. Comment est-il devenu possible? Descartes, d'après Leibniz, a fourni la plus grande partie de la technologie requise pour la formation de ce concept el cependant a délibórément reculé devant tout ce qui pouvait ressembler à notré concept de démonstration. Je soutiens que Descartes, dans son rejet implicite de notre idée de démonstration, et Leibniz dans son attachement excessif à elle, essaient tous les deux de remédier à un malaise fordamental dans l'épistémologie du dix-septicme siècle. Je parle d'un malaise plutớt que d'un problème ou d'une diflieulté, car il n nétait pas formulé et n'ètait péut-ètre pas formulable. (...) l.eibriz était sûr que la vérité mathéntalique est constitứe par la démonstration, alors que Descartes estimait que les conditions de la vérité n'ont rien à voir avec la démonstration. Nous reconnaissons ces doctrines qui s'altrontent dans unz bonne partie de la philosophie des mathérmatiques de l'époque moderne. La manière dont les deux figures historiques ont déterminé un bon nombre de nos préoccupations les plus récentes n'est pas passée inaperçue: Yvon Belawal connnence délibérément son livre importanı sur Leibniz et Descartes par un long chapitre intitulé "Intuitionnisme et formalisme". Il y a la une quantité d'autres parallèles à tracer. Je trouve que ce n'est pas une coincidence, car je suis tourmenlé par une conjecture, à la fois non démontrée et dépournue d'originalité, selon laquelle l'"espace" d'un problème philosoplique est largement fixé par les conditions qui l'ont rendu possible. Un problème est individué uniquement par l'utilisation de certains concepis, et les conditions de l'émergence de ces concepts déterminent de façon presque embarrassante ce qui peut étre lait avec eux. Les solutions, les contre-solutions et les dissolutions sont élaborées dans un espace dont les propriétís ne sont pas reconnues, mais doml les dimensions sont aussi solidement assurées qu'tlles sont inconnure.'
\end{abstract}

A la fin de son article, llacking fait un rapprochement à la fois suggestif et surprenant, qui est destiné à montrer à quel point les discussions contemporaines continuent ellectivement à être déterminées par les mêmes concepts et, plus préciseśnent. par les conditions spéciliques dans lesquelles les concepts en question sont apparus:

\begin{abstract}
"Prencz, par exemple, la plus nouvelle en apparence et également la plus passionnement disparate des contributions, les Remarques sur les fondements des malhématiques de Wittgenstein. Il nous invite à

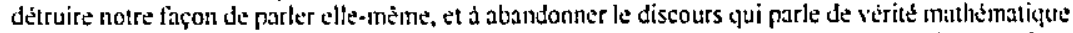
et de connaissance des mathématiques et de leurs objets. On nous demande d'essayer un langage dans lequel les mathematiqutes ne sont pas 'vraies', nos découvertes ne sont pas unk 'comaissance' et les 'objets' ne sont pas des objets. En dipit de cette tentative etrange et diconcentante pour se debarrasser de toutes ces notions hisiteses, Wittgenstein tinit avec un dilemme gut est essentiellement leibrizianocartisien. D'un còté, il suggère, de lo façon la plus radicale qui soit, que la 'vérité madhématiqute est constittúe par la dèmonstration, et, de l'autre, il est obsédé pardes intuitions qui sont justement celles qui ont tellement impressionné Deseanes. Pratiquement personne ne pense qu'il a réussi une synthese
\end{abstract}

1 "Leibniz and Descartes: Proot and Etemal Trutls", in $\Lambda$. Kenny (od.). Rationalism, Empiricism and lderlism, British Academy Lectures on the History of Philosoplyy, The Clarendon Press. Oxford, 1986, p. 47. 
de ces notions. II y a une raison à cela. II rejette ce tryptique ancien, véritê, connaissance et objets, mais travaille dans l'espace crés par cette période antúrieure et est conduit à utiliser les concepts créds à ce moinent-lí pour la résolution de tout autres problèmes, et qui sont cntravés par le besoin que l'on a d'eux pour résoudre ces autres problèmes. La 'bouteille a mouchı's' a rę̧u sa forme de la préhistoire, et seule l'archéologie peut rendre visible celte forme" (ibid., p. 60).

Ce qui est, si l'on veut, “leibnizien", dans l'attitude de Wittgenstein, est le fait de considerer non seulement la vérité, mais mème la signification de la proposition madıématique comme liées intrinsèquement à la démonstration; et ce qui est "cartésien", aux yeux de Hacking, est, je suppose, le fait qu'il peut donner l'impression de remplacer la doctrine de la création divine des vérités étemelles par l'idée que les vérités nécessairtes sont en réalité crées par nous, en ce sens qu elles sont acceptées et instituées comme règles au terme du processus de la démonstration et pourraient éventuellement ėtre différentes si nous étions disposés à utiliser ou avions des raisons d'utiliser un concept différent de ce qui peut être reconnu comme une démonstration correcte. Si la vérité mathématiqué est essentiellement le produit de la démonstration, c'est, semble-t-il, la notion de vérité elle-même qui devient problématique, puisq̨ue la démonstration, telle que la comprend Wittgenstein, effectue une détermination de sens et ne nous conduit pas à la reconnaissance d'un fait mathémalique qui pourrait être consideré comme réalisé indépendamment d'elle.

Je ne me propose pas, bien entendu, de discuter ici la question de savoir si la description que donne Hacking du projet de Wittgenstein, dans les Remarques sur les fondemenls des math'matiques, correspond ou non aux intentions réelles de l'auteur, et pas non plus celle de savoir si nous avons besoin de l'archéologie, au sens de Foucault, pour comprendre les raisons de l'écleec d'une tentative comme la sienne, qui provient, selon Hacking, du fait que nous continuons à traiter et sommes d'une certaine façon contraints à traiter des problèmes comme ceux qui se posent à nous aujourd'hui en philosophie des mathématiques dans des termes et à l'aide de concepts qui ont été produits initialement pour résoudre des problèmes d'une tout autre nature. Ce qui m'intéresse n'est pas la la manière dont ce que Hacking appelle l'"espace" du probleme ou de la problématiquc philosophiques, tel qu'il le décrit, peut continuer à déterminer et éventuellement à hypothesquer les tentatives de solutions actuelles, mais plutôt la description qu'il donne de cat espacc.

Il y a au moins trois raisons pour lesquelles la présentation qu'il donne des positions respectives de Descantes et de Leibniz sur la question de la démonstration peut sembler à première vue très surprenante:

1) Descartes a dit et répété que le modèle à imiter pour avoir une chance de parvenir à la vérité en philosophic śtait celui de la démonstration mathématique. II ne s'agissait de rien de moins, pour lui, que de procurer à la métaphysique des démonstrations qui soient, comme il le dit. "aussi mathématiques et évidentes" que celles des mathématiques elles-mêmes. Il est pour le moins diflicile, dans ces conditions, d'accepter l'idec qu'il ne savait pas ce qu'est une démonstration dans notre sens et ne croyait pas que la vérité puisse avoir un lien essentiel avec la démonstration. Comme le dit Guéroult: "La philosophie cartésiente se veut rigoureusement démonstrative. Son auteur ne cesse de répéter qu'il suit l'ordre des géomètres, qu'il n'y a pas de bonne démonstration en philosophie qui ne soit pas mallématique, que son auvre ne peut être saisie par ceux qui n'ont pas l'esprit mathématique. Il tombe, par conséquent, sous le sens qu'on doit s'efloreer de comprendre celte plilosophie par ses dímonstrations, et ces démonstrations selon leur esprit mathématique."2 Puisque Descartes dit, dans l'Abrégé des Méditations:

2 Martial Gubroult, Descartes selon l'ordre des raisons, Aubier-Montaigne, Paris, 2 cune édition, 1968, tone l, p. 12. 
qu'il a "tâché de ne rien écrire dans ce traité, dont je n'eusse des démonstrations très txactes", 3 on est, semble-t-il, autorisé à supposer qu'il savait ou, en tout cas, croyait savoir ce qu'est une démonstration exacte. Mais, en mêmes temps, il est clair que le problème ne peut pas être de savoir si Descartes fournit ou non en philosophie des démonstrations qui peuvent être considérées réellement comme mathématiques selon nos critères (et même déjà ceux de Leibriz, qui est effectivement on ne peut plus sceptique sur le caractère réellement démonstratif de la démarche cartésienne), mais plutôt, étant donné que les démonstrations qu'il propose sont pour lui indiscutablement mathématiques, de savoir ce qu'il entend exactement par "démonstration" dans les mathématiques elles-mêmes et par "mathématique" dans l'expression "dímonstration mathématique".

2) Comme le remarque Hacking lui-même, si on compare les façons de faire respectives de Descartes et de Leibniz, on se rend compte que ce n'est pas, de façon générale, le deuxième mais plutôt le premier, qui se montre, dans la pratique, le plus strict et le plus respectueux des exigences de la démonstration exacte. Leibniz, qui est souvent trop rapide, étourdi ou négligent, n'est pas forcíment un modèle d̀ imiter, lorsqu'il s'agit de produire des démonstrations formellement correctes. Descartes, qui méprisait le formalisme, est, en revanche, presque toujours formellement correct.

3) Il est à première vue étrange d'attribuer à Descartes l'idée que la démonstration est sans pertinence réelle et sans importance pour la véritś et d'approuver en méme temps la façon qu'a Belaval de rattacher Descartes à une tendance que l'on peut qualifier d'“intuitionniste". au sens large, alors que Leibniz devrait être considéré au contraire, pour sa part, comme un précurseur direct du formalisme. Car ce qui caractérise l'intuitionnisme en philosophie des malhímatiques semble être justement une façon de lier la question de la vérité à cêlle de la démontrabilité, qui interdit de donner un sens quelconque à l'idée de propositions mathématiques qui pourraient être vraies ou fausses, en dépit du fait que nous ne sommes pas et ne serons peut-être jamais en mesure de les démontrer ou de les réfuter. Pour un intuitionniste, être vrai, lorsqu'il est question de la vérité d'une proposition mathématique, ne peut signifier autre chose qu'être démontré ou, en tout cas, démontrable. Et cela semble impliquer clairement qu'il est impossible de parler d'une indépendance de la vérité mathématique par rapport à la démonstration. L'idée d'une indépendance de cette sonte semble être, au contraire, ce qui caractérise la position du réalisme mathématique et ce que les intuitionnistes luj reprochent. Pour le réalisme, la rélation qui existe entre la vérité et la démontrabilité est une relation externe: il est tout à fait concevable qu'il existe des propositions mathématiques qui sont vraies. en dépit du fait qu'elles ne seront peut-ètre jamais démontrées. Pour l'intuitionnisme, la relation est, au contraire, interne et essentielle: une proposition mathématique vraie est une proposition que nous sommes en mesure de vérifier, autrement dit de démontrer.

La réponse à ces trois types d'objection possibles ne présente, heureusement, pas de difficulté majeure. Ce que Hacking suggère n'est, bien entendu, pas littéralement que, pour Descartes, la démonstration n'avait pas d'utilité réelle en mathématiques ou en philosophic ou qu'il ne considérait pas comme essentiel d'être capable de distingutr entre une démonstration correcte et une démonstration erronce, mais seulement qu'il ne considérait pas comme indispensable ou même simplement utile de disposer d’un concept précis et explicite de ce qu est une

3 CEnres de Descartes, publiées par Charles Adam et Paul Tannery, Vrin, Paris, 1996, tome VII, p. 12-13 (abregé dorénavant AT). Descartes: Oewres et Lelles, Textes présentés par André Bridous, Bibliothèqu de la Pléiade, Gallimard, Paris, 1953, p. 262 (abrégé dorénavant P). 
démonstration et, en tout cas, n'a anticipé ni de près ni de loin la conception de la démonstration qui est devenue dominante aujourd'hui, alors que le concept leibnizien de la dímonstration est déjà presque exactement le nôtre. Dans les Regulke, Descartes après avoir expliqué que nous parvenons à la connaissance des choses par deux chemins, qui sont l'expérience et la déduction, ajoute: "Il faut noter (...) que les expériences sont souvent trompeuses, mais que la déduction ou la simple inférence d'une chose à partir d'une autre, peut sans doute ètre omise si on ne l'aperçoit pas, mais ne saurnit être mal faite même par l'entendement le moins capable de raisonner. Mais, pour y réussir, je trouve d'une médiocre utilité ces chaines, par lesquelles les dialecticiens pensent gouverner la raison humaine, bien que je ne nie pas qu'elles soient excellentes pour d'autres usages. En effet, toutes les erreurs où pensent tomber les hommes (et non les bêtes, bien entendu) ne proviennent jamais d'une mauvaise inférence, mais seulement de ce qu'on adnet certaines expériences peu comprises ou qu'on porte des jugements à la légère ou sans fondement" (Regulae ad directionem ingenii, AT, X, p. 365; P, p. 41). Puisque Descartes pense que ce qui caractérise les infërences est le fait de neâtre pas faites du tout ou, lorsqu'elles sont faites, de l'être correctement mème par les esprits les moins douis et les moins éduqués, il est tout à fait logique de sa part de considérer que la formulation d'un ensemble de règles formelles qui auraient pour but de déterminer à quelles conditions une déduction peut être considérée comme valide n'est pratiquement d'aucun secours, lorsqu'il s'agit de maintenir la raison sur le chemin de la vérité. Comme il l'explique dans l'Entretien avec Burman (AT, V, p. 175; P, p. 1397), non seulement la logique (ou, en tout cas, la dialectique, qui traite formeltement de toutes choses en nous détournant de la nature méme des choses) ne constitue pas une aide pour ce qu'il appelle le "bon sens", mais elle a plutôt pour eflet de le pervertit ou de le detruire. Ce qui importe n'est pas que nous disposions d'un concept savant de la déduction, dont les logiciens seraient cliargés de déterminer les caractères, mais uniquenent que nous soyons assurés de pouvoir reconnaître une déduction correcte, lorsque nous en rencontrons une. Et, sur ce point, Descartes n'a manifestement aucune inquićlude.

Il ne se contente pas d'affürmer que l'évidence et la certitude de l'intuition "ne sont pas requises seulement pour de simples affirmations, mais aussi pour toute espece de raisonnement" (Regulc, AT, X, p. 369; P, p. 44). Elles sont aussi, en un certain sens, suffisantes. Lorsqu'on effectue un modus ponens, il faut, bien entendu, voir par intuition non seulement que " $A$ " et "Si $A$, alors $B$ " sont vrais, mais également que " $B$ " suit nécessairement de ces deux propositions. Mais c'est une chose qui peut être vue aussi facilement et aussi súrement que la vérité de "A" ou de "Si A, alors B". On est, bien entendu, oblige de faire, et c'est une chose particulièrement évidente dans le cas les malhématiques, une diffërence entre ce qui peut être connu avec certitude et ce qui est ćvident, puisque Descartes admet que, si les premiers principes peuvent être connus par intuition, les conséquences éloignées ne peuvent l'être, en revanche, que par deduction. Si on prend, par exemple, le cas d'un thiorème caractéristique de la tléorie des nombres, comme par exemple le thcorime de Fermat, ce qüil faut dire est, semble-t-il, qu'il est connu avec certitude, mais certainement pas qu'il est ćvident. Il serait, en effet, difficile de pritendre que la démonstration nous conduit dans tous les cas de ce genre non seulement à la certitude, mais également à la perception claire et distincte de la vérité de la conclusion. Ou, en tout cas, si une perception de cette sorte est possible, elle ne semble, justement, pas pouvoir être séparée de la perception claire et distincte des étapes du processus démonstratif qui a conduit à la reconnaissance de la vérité de la proposition. On peut dire de la plupart des propositions mathematiques que nous reconnaissons comme vraies que nous savons avec certitude qu'elles sont vraies, parce que nous savons qu'elles ont ćté démontrées, mais on ne voit pas comment 
la reconnaissance de la vérité pourrait ètre transformée en une perception claire et distincte ou. en tout cas, en une chose qui se rapproclie sultisamment de ce cet ideal, sans que cela implique la réeffectuntion de ce que Descartes appelle "le mouvement continu et ininterrompu de la pensée" qui, dans le cas idéal, nous conduit, par un chemin qui reste fondamentalement celui de l'intuition, des prémisses à la conclusion de la démonstration.

Cela pose évidemment un problème pour une interprétation comme celle de Hacking. Mais ce qui est clair est que Descartes n'accorde eflectivement pas une importance extrêtne à la diţěrence qui peut être faite entre ce qui est connu directement par intuition et ce qui est connu seulement par deduction. La distinction est pour lui, de toute évidence, relative et llacking n'hésite pas à dire qu'elle est méme essentiellement psychologique. Un homme peut avoir besoin de déduire, là où un autre intuitionne et ce qui est connu à un moment donné par déduction peut à un autre moment être intuitionne. Descartes souligne lui-mème qu'*il y a des choses qui sont ainsi connues sans preuves par quelques-uns, que d'autres n'entendent que par un long discours et raisonnement" (AT VII, p. 163-164; P, p. 393). Le lecteur moderne a tendance à assimiler l'intuition à la reconnaissance de la vérité des aviomes et la déduction à celle de la vérité des théorèmes; mais, comme le remarque Hacking, c’est déjà voir les choses à la laçon de Leibniz, qui a, pour sa part, un point de vue explicitement axiomatique sur la question, et non de Descartes, même si, pour les raisons que je viens d'indiquer, il ne va pas de soi que la connaissance de la vérité des lléorimes puisse, aussi bien que celle de la vérité des axiomes, être une chose qui mérite réellement d’ètre appelée une perception claire et distincte de la vérité. Ce qui est clair, est que, pour Leibniz, il n'y a qu'une espèce de propositions qui puissent être connues salls démonstration, qui est déterminée objectivement et la même pour tout le monde; et ce sont les axiomes proprement dits, qui sont toujours des identités explicites. Toutes les autres propositions, y compris les axiones usuels, qui, la plupart du temps, ne sont pas de cette forme, sont démontrables et doivent en principe être démontrés. Ce n'est évidenument pas du tout le point de vue de Descartes.

La supériorité de l'arithmélique at de la géométrie ne réside en aucune façon. pour lui, dans le fait qu'elles ont pris soin de se doter d'un appareil déductif précis et sophistiqué qui les protègerait plus efiicacement que les autres sciences contre le risque de l'erreur, mais dans le fait que "seules elles traitent d'un objet assez pur et simple pour n'admettre absolument rien que l'expérience ait rendu incertain, et qu'elles consistent tout entières en une suite de consíquences déduites par raisonnement" (ibid.). Leur avantage est done de n'être menacées ni par le risque d'erreur que représentent les expériences mal comprises ni par celui que pourrait repr:senter le passige de propositions reconnues initialement conme certaines à d'autres cjui en dérivent, puisque celui-ci s'elfectue entièrement par déduction, c'est-à-dire, pour Descartes, d'une façon qui exclut en principe la possibilité de se tromper. Il dit de l'arithnétique et de la géomítrie qu'elles sont les plus faciles et las plus chaires de toutes les sciences, "et leur objet est tel que nous le désirons, puisque, sauf par inattention, il semble impossible à l'homme d'y commettre des erreurs" (ibid.).

On peut penser qu'il s'agit là d'une conception extraordinairement optimiste. Le mathématicien clevronné qu était Descartes ne pouvait vraisemblablement pas ignorer la lacilité avec laquelle des démonstrations incompletes ou meme illusoires peuvent être acceptés et même acceptées pendant longtemps comme parlaitement probantes dans les mallématiques elles-mémes. Mais, pour lui, l'erreur ne provient jamais dans ce domaine d'un défaut de connaissance, par exemple de l'ignorance ou d'une maitrise insuflisante des règles lormelles qui gouvernent la praticue du raisonnement déductif, mais toujours uniquement de l"inattention. Si 
l'arithmétique et la géométrie sont les sciences à la fois les plus faciles et les plus sûres, e'est parce que rien d'autre que le "bon sens", c'est-à-dire la capacite de distinguer le vrai et le faux par le seul usage de la lumière naturelle - une aptitude qui, à la diflèrence de la familiarité très inégale que peuvent avoir les lommes avec une discipline technique ct même ésotérique comme la logique, est également répartie entre eux-, n'a besoin d'y intervenir. Peirce soutient qu'autant le recours à une théorie du raisonnement élaborée et sophistiquée est inutile et même néfaste dans les questions ordinaires, autant il est indispensable dans la métaphysique. -Une Logica Ulens, écrit-il, comme la mécanique analytique qui réside dans les nerfs du joueur de billard, est ce qui répond le mieux aux usages familiers. " Descartes pense que c'est à peu près le contraire qui est vrai: la métaphysique, comme, du reste, les mathénatiques ellesmêmes, n'ont pas besoin d'une Logica docens, mais seulement d'une Logica Utens, qui est constituée en l'occurrence simplement par le bon sens orienté et discipliné uniquement par les règles de la méthode.

En ce qui concerne la difficulté que pourrait sembler présenter une comparaison de la position de Descartes avec celle des intuitionnistes, il suffira de remarquer que, lorsque les intuitionnistes soutiennent qu'être, pour un objet mathématique, veut dire être construit ct être vrai, pour une proposition mathématique, veut dire être démontré, ce qu’ils entendent par "construction" et par "démonstration" n'a rien à voir avec l'exécution d' une procédure formelle ou même d'une procédure qu'il pourrait ètre intéressant d'essayer de formaliser. Pour Brouwer, il est parfaitement futile et incongru d'essayer de codifier a priori et une fois pour toutes dans des règles formelles l'ensemble des démarches qui sont susceptibles de nous conduire à la reconnaissance d'une vérité mathématique. Brouwver résume à un moment donné l'opposition qui existe entre lui et les formalistes en disant que, pour l'intuitionnisme l'exactitude réside dans l'esprit, alors que, pour le formalisme, elle se situe sur le papier. C'est une caractérisation qui pourrait être utiliséc dẻjà pour exprimer le désaccord fondamental qui existe entre Descartes et Leibniz sur la question de la démonstration. Descartes considere que le critère ultime de la vérité est la perception claire et distincte et cela entraine chez lui une méfiance du même genre que celle que l'on retrouvera plus tard chez. Brouwer à l'égard des prétentions de la logique. Brouwer considère que la logique ne constitue en aucune façon l'autorité ultime sur la question de la vérité mathématique. Comme le dit le titre de l'un de ses articles, "les principes logiques ne sont pas sûrs" et, s"ils sont appliqués aux mathénatiques, considérées sous leur aspect uniquement symbolique et en l'absence de la contre-partie et de la garantie constitućes par la présence d'une évidence proprement mathématique qui leur correspond à chaque fois, ils sont parfaitement susceptibles de nous conduire dans certains cas à l'acceptation de propositions mathématiques fausses.

Descartes ne reconnaît pas davantage à la logique la position d'arbitre suprème, en matière de vérité, mathénatique ou autre. Comme le dit Belaval, chez lui, "les principes de la

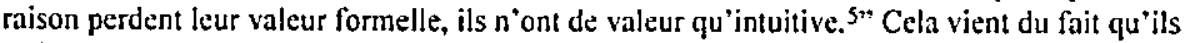
ne sont pas perçus eux-mèmes intuitivement, mais seulement en liaison, ou plutôt comme liaisons entre les termes qu'ils conjoignent. Les notions communes ne sont perçues distinctivement que lorsqu'elles lient entre elles des idées distinctes et ne constituent en dehors d'elles

4 Charles Sanders Peirce, Reasoning and the Logic of Things, edited by Kenneth Laine Kether. with an introduction by Kenneth Laine Kutmer and Hilary Putnam, Harvard University Press, Cambridge, Mass., London, Englahd, 1992, p. 109.

5 Yvon Belaval, Leibni= critique de Descarses, Gallimard, Paris, 1960, p. 63. 
que des énonciations purement verbales sans contenu ni vérité. Les principes formels de la logique ne permettent pas à la raison d'instaurer des relations entre les idées, elle ne fait que constater leur existence, lorsqu’elle considere les idées avec une attention suflisante.

\section{L'intuition et le formalisme}

Rien n'est donc plus étranger à Descartes que l'idểe qu"une formalisation des principes et des règles du raisonnement logique pourrait représenter un progrès essentiel du point de vue de la rigueur dans les mathématiques ou dans la métaphysique. Car, pour lui, la correction du raisonnement ne peut stre garantie, justement, que par une attention suflisante et constante au contenu lui-meme, et certainement pas par la séparation rigoureuse de la forme d'avec le contenu et le respect de règles formelles de quelque nature que ce soit. Leibniz, qui est considéré souvent a juste titre comme le véritable père de la logique moderne, a une conception bien différente et incomparablement plus proche de celle qui a inspiré les eflorts des logiciens contemporains. "La droite raison est, cerit-il, un enchaînement de vérités, la raison corrompue est mêlée de préjugés et de passions, el pour discerner l'une de l'autre, on n'a qu'à procéder par ordre, n'admetire aucune these sans preuve, et n'admettre aucune preuve qui ne soit en bonne forme selon les règles les plus vulgaires de la Logique. On n'a point besoin d'autre critérion ni d'autre juge des controverses en matière de raison." ${ }^{6}$

Belaval note que: "Contre la tradition d'Aristote, maintenue par l'Ecole, Descartes attend de la méthode une conversion de l'esprit, il n'en attend pas des techniques appropriées aux diffërentes matières" (op. cit., p. 27). On pournit croire qu'en mathématiques découvrir la vérité signilie démontrer et que la mèthode qui garantit l'accès à la vérité est celle qui consiste à démontrer rigoureusement tout ce qui peut l'štre à partir de principes suffisamment évidents pour n'avoir pas besoin d'âtre eux-mémes dimontrés. Or c'est justement ce que pense Leibniz et non Descartes, et ce que Leibniz reproche à Descartes de n'avoir pas fait dans sa philosophic. Pour celui-ci, à partir du moment oú nous sommes parvenus à une perception claire et distincte de la vérité d'une proposition, il importe peu que celte perception soit obtenue directement par une intuition, plutót qu'indirectement par un processus de dsduction, et nous ne pouvons pas compter sur un systeme de règles formelles pour garantir la transmission non seulement de la vérité, mais également de la perception claire et distincte de la vérité, des premisses a la conclusion dans un raisonnement. Car ce qui compte est uniquement in perception claire et distincte, qui résulte à chaque fois de la présence innmmédiate et de la transparence du contenu lui-même, et non l'application de techniques de démonstration dans lesquelles le symbolisme lui-mème travaille en quelque sorte à notre place. Comme le dit Belaval: -... Pour Deseartes, contre le formalisme, qui laisse la mison oisive, la mílhode doit âtre d’abord un exercice de la volonté qui rende l"attention active" (ibid., p. 28). Elle doit être aussi une laçon de mobiliser le plus eflicacement possible les ressources de la volonté dans l'exercice du jugement, qui, pour Descartes, est et doit rester une operation du vouloir, et non de l"intellect, alors que la conception formelle du raisonnement tend au contrate à dissocier complètement le processus qui aboutit au jugement de l'intervention de la volonte.

Leibniz soutient que la dimonstration est valide en venu de sa fome, et non de son contenu. Elle est constituce d'une suite de propositions qui commence par des identités explicites

\footnotetext{
6 Essais de Theodice, Clironologic et introduction par 3. Brunscluvig, Garnier-Flammarion, Paris, 196\%, p. 86.
} 
totales ou partielles, c'est-à-dire des propositions de la torme " $A$ est $A$ ", "AB est $A$ ", ctc., et dont chaque proposition est tiré d'une des précédentes par une application du principe de substituabilité des termes identiques salva veritate. La dimonstration, au sens strict, ne requiert en tout et pour tout que trois choses: des axiomes identiques, des définitions et le principe qui autorise à remplacer l'un par l'autre dans toute proposition un terme délini et sa définition. Leibniz fait certainement preuve d'une confiance exagére, lorsqu'il allirme qu“il est possible de démontrer avec des moyens aussi limités toutes les propositions qui sont vraies, et en tout cas toutes les vérités mathématiques. C'est presque certainement à lui que Gödel a emprunté le concept de ce qu'il appelle l'analyticité au sens étroit, c'est-ì-dire le sens purement formel auquel les axiomes et les thiorèmes d'un systeme comme celui des Principia Mathematica peuvent être dits analytiques si et seulement si les termes qui y fïgurent peuvent être délinis (explicitement ou par des règles qui permettent de les éliminer des propositions qui les contiennent) d'une manière telle qu'ils deviennent des cas particuliers de la loi d'identité et les propositions rífutables des négations de cette loi. ${ }^{7}$ Or on sait aujourd hui qu“il est possible de demontrer que mème la thcorie des nombres entiers n'est pas analytique en ce sens-là, si on exige des règles d'élimination qu'elles permettent d'ellectuer dans tous les cas l'élimination en un nombre fini d'ćtapes.

Mais il ne peut y avoir, en revanche, aucun doute sur le lait que le concept de démonstration que propose Leibniz est rigoureusement formel et pour l'essentiel identique au nôtre. La démonstration des propositions nécessaires, qui sont les seules que nous puissions eflectivement démontrer, se présente, clez lui, comme une suite linie de transformations purcment formelles effectuées sur des signes, elle peut être assimilè entièrement à un calcul et testée quant à sa correction par le mème genre de procédure mécanique qu un calcul effectué sur des nombres, ou plutôt sur des signes numériques. Leibniz dispose d'une notion de dímontrabilité qui est rigourcusement sy'ntaxique en ce sens qu'elle repose sur les deux principes suivants: 1) Toutes les propositions qui sont distinguées par une certaine propriété purcment structurale (les axiomes explicites) sont vraies, et 2) Toutes les propositions qui résultent de l'application de certaines operations formelles à des propositions déjà reconnues comme vraies sont également vraies. Descartes réve, comme le dit Belaval, d'une déduction qui ne serait rien d'autre qu une intuition continuéc. Leibniz pense que la scule lorme d’intuition dont nous avons besoin ici est l'intuition concrète des signes.

Comme le remarque Hacking, il ignore le problème que pose l'équivalence de la notion syutaxique de démontrabilité formelle et de la notion sémantique de vérité nécessaire, comprise, chez lui, comme stant celle de vérité dans tous les mondes possibles, autrement dit le probleme de la complétude; et il lait de cetle équivalence une simple délinition en postulant que toute proposition vraie doit âtrégalement démontrable, les propositions nécessaires par un nombre lini et les propositions contingentes par un nombre infini de substitutions définitionnelles. "De façon géncrale, écrit-il, toute proposition vraie (qui n"est pas identique ou vraic par soi) peut être démontrée a priori à l'aide d"axiomes ou de propositions qui sont vraies par soi et à l'aide de délinitions ou d'idés."8 Que toute proposition vraie doive être également démontrable ne constitue pas un problème pour lui, puisque c'est une conséquence immédiate du principe de raison suftisante, ou plutôt une autre façon de formuler le principe lui-méme.

7 Cf. Kur Godal, "Russell's Mathematical Logic" (194f), in P. Benacerraf and hlilary Putnsin (eds.), Philosoply, of Mtahtematics, Selected Essays, 2nd. edition, Cambridge University l'ress, Cambridge, 1983, p. 467.

8 Philosophische Schrifien, herausgegeben von C. 1. Gerhardt, Georg Olms, Ilildesheim, 1966, VIt, p. 300. 
Une proposition vraic qui ne serait pas démontrable serait une proposition dont la vérité est sans fondement et pour laquelle nous ne saurions tout simplement pas ce que signilie le hait d'être vraie.

Rien n'illustre plus clairement la dépendance fondamentale de la vérité par rapport à la démonstration, chez Leibniz, que le fait que la conpréhension complète de la vérité de la proposition ne puisse étre constituce que par la demonstration elle-mème. Nous ne comprenons done pas complétement les vérités contingentes, puisque nous avons besoin de recourir à d'autres sources que l'analyse des concepts pour reconnaitre leur vérité. Nous n'avons la plupart du temps de la vérité des propositions de cette sorte qu'une connaissance que l'on peut appeler factuelle ou historique et qui n'est pas, comme celle des vérités nécessaires, purement conceptuelle, en dépit du fát que le fondement ultime de leur vérité ne dépend, lui aussi, que du contenu des concepts qui y figurent. Il va sans dire que Leibniz est, sur ce point, le contraire d'un sceptique et il ne conteste aucunement qu'une multitude de propositions empiriques puissent être connues avec une certitude qui est tout à fait suffisante. Mais il y a, pour lui, une diflërence essentielle entre connaitre le simple tait de la vérite et connaitre, en outre, la raison de la v'érité. On retrouve donc chez lui une distinction analogue à celle que fait Descartes eutre le fait de savoir qu'une proposition est vraic ou, comme il le dit, de toucher sa vérité et le fait de réussir à la comprendre ou à l "embrasser réellement dans son esprit: "... Comprendre, c'est embrasser de la pensée, mais pour savoir une chose il suflit de la toucher de la pensée" ( 1 . Merseme, 27 mai 1630, AT, 1, p. 152; p. 938). Il y a, pour Descartes, une multitude de propositions tris importantes qui sont telles que nous avons une perception claire et distincte de leur véritś, sans être pour autant en mesure de les comprendre. La distinction entre savoir et comprendre est, chez Leibniz comme chez lui, liéce a l'intervention de l'inlini. Mais, pour Descartes. il s'agit de l'infini de la toutt-pussance divince et pour Leibniz de ce qu'il appelle l'inlini dans les raisons. qui est la caractéristique de toutes les propositions que nous ne sommes pas en mesure de démontrer, et donc de comprendre complètement. La différence entre connaitre la vérité et la comprendre ne signilie évidemment pas du tout la meme chose dans les deux cas: l'indini dans les raisons na rien à voir, chez Leibniz, avec l'impossibilite de se faire une ide de la raison. Pour lui, nous savons a priori que ce que nous ne comprenons pas est en principe comprélensible par un processus qui n'est pas fondamentalement difürent de celui que nous utilisons dans la dímonstration des propositions nécessaires et que nous sommes simplement incapables. en l'occurrence, de mener à son terme. Ce qui nous empéclte de comprendre récllement les desseins et l'auvre du créateur est simplement le fait que notre entendement limite ne dispose pas des moy'ens de calcul qu'il a utilisés pour déterminer ce qui itait le meilleur et le choisir. Pour Descartes, sur ce qui dépasse la raison humaine, on ne peut rien aflirmer ou nier. Pour Leibniz, nous pouvons avoir, au contraire, une idie précise des contraintes auxquelles ont it: soumises, dans la création, l'entendement et la volonté de Dieu, les régles de la logiçue, pour ce qui est de l'entendement, et la nécessité, qui n'est assurément pas logique, mais seulement morale, de choisir le meilleur, pour ee qui est de la volonté, ha nécessité de le choisir, après lavoir reconnu, et non pas, comme clez Descartes, la liberté de le decréter souverainement. En subordonnant comme il le fait non seulement la nécessité morale, mais également la nécessité logique, aux decrets de la toute-puissance divine, Descartes introduit préciséınent une distance que Leibniz considere comme tout à hait exessive et inacceptable entre ce que nots pourons savoir et mème savoir avec certitude et ce que nous pouvons, en outre, comprendre.

Comme le dit Marion à propos de Suarez: "L indépendance des vérités éternelles ne s impose (...) à Dieu gu en vertu de l'univocité de la vérits: la même identité logicue (done les 
mémes contradictoires) s'impose à Dieu comme aux esprits créés. L'univocité du savoir constitue la condition épistémologique radicale de l'indépendance des vérités éternelles." Leibniz. est, sur ce point, du côté de Suarez et de tous ceux qui pensent que la connaissance que nous avons des vérités de raison n'est pas obtenue d'une autre maniere que celle que Dieu en a et pas différente d'elle dans son principe, et que nous savons très bien, en outre, en quoi consiste la connaissance complète que Dieu a des vérités de fait elles-mêmes et pourquoi elle nous est interdite. II n'y a pas pour Leibniz deux fał̧ons complètement diff̈̈rentes de savoir: savoir en connaissant complètement ou partiellement les raisons, c'est-à-dirc en comprenant, et savoir d'une façon qui reste constitutivement et délinitivement en deçà de la connaissance des raisons et interdit même de s'interroger sur ce qu'elles peuvent ètre, autrement dit qui est sans rapport avec le savoir que Dieu possède des mêmes choses.

\section{Tradition et innovation: Leibniz et ses prédécesseurs}

Hacking dit que la compréhension leibnizienne de la nature de la dimonstration n'a pas vaiment existé avant Leibniz. Mais la première chose à remarquer sur ce point est certainement que Leibniz n'a aucunement, pour sa part, l'impression de proposer une innovation radicale. II est persuadé de revenir simplement à une conception qui était déjà celle d'Aristote et des Scolastiques, c'est-à-dire la conception selon laquelle un raisonnement logique ne peut être contraignant que vi formae, en veru de sa forme et indépendanment de son contenu, ce qui implique précisément que sa validité doit pouvoir être testée par des procédures purement formelles et sans recours à l'intuition. "Raisonner machinalement, icrit Jean Laporte, c'est bien l'idéal de la logique scolastique comme de toute logique formelle" (cité par Belaval, op. cit., p. 35). C'est aussi l'idíal de Leibniz, qui ne voit rien de dégradant pour l'esprit dans cette fişon de se prémunir contre l'erreur. De son point de vue, ce n'est pas lui qui introduit une idée révolutionnaire de ce qu'est la démonstration, mais Descartes qui se singularise en adoptant une conception qui se distingue radicalement de celle de tous ses grands prédícesseurs. "On me demandera, écrit-il, où est donc ce beau moyen qui nous peut garantir des clutes? J'ai quasi peur de le dire: cela paraîtrait trop bas, mais enfin je parle à $V$. $A$. qui ne juge pas des choses par l'apparence. C'est en un mot, de ne faire des arguments qu'in forma. Il me semble que je ne vois [que] des gens qui s'écrient contre moi et qui me renvoient à l'école. Mais je les prie de se donner un peu de patience, car peut-être ne m'entendent-ils pas; les arguments in forma ne sont pas toujours marqués au coin de Barbara Celarent. Toute démonstration rigoureusc qui n'omet rien qui soit nécessaire à la force du raisonnement est de ce nombre, et j'ose bien dire qu'un compte de receveur et un calcul d'analyse est un argument in forma, puisqu'il n'y' a rien qui y manque, et puisque la forme ou la disposition de tout ce raisonnement est cause de l'évidence. Ce n'est que la forme qui distingue un livre de comptes fait selon la pratique qu'on appelle communement italienne (dont Stevin a fait un traité tout entier) d'un journal confus de quelque ignorant en matière de négoce. C'est pourquoi je soutiens qu atin de raisonner avec évidence partout, il faut garder quelque formalité constante. It y aura moins d'éloquence et plus de certitude" (Phil. Schr., IV, p. 295).

Comme l'a fait remarquer Scholz, Leibniz est un des premiers et peut-être le prenier à avoir reconnu clairement l'essence de ce qu'on appelle un calcul: "Un calcul au sens mathématique est un dispositif de règles de transformation qui permettent de remplacer des opé-

9 Jean-Luc Marion, Sur la théologie blanche de Descartes, P. U. F., Paris, 1981, p. 69. 
rations de penséc effectuies sur des objets mathématiques quelconques par des transformations mecaniques de certaines suites de signes prépares pour cette fïn." ${ }^{10}$ Leibniz a, du mìme coup, reconnu clairentent qu'il était possible, moyennant une notation appropriée, de calculer sur bien autre chose que des nombres ou des grandeurs (ou des objets mathématiques en général), par exemple sur des concepts ou des propositions. Comme en timoigne la comparaison qu'il hit de la démonstration avec un calcul de comptable, il est sans doute le premier à allirmer aussi clairement que l'essence de la démonstration réside entièrement dans ce que l'on peut appeler son caractère formel-computationnel et il considère qu'il doit être possible de donner dans tous les cas à la procédure démonstrative, y compris lorsqu'elle opère dans le domaine de la métaphysique, la forme d'un calcul, au sens indiqué.

II est done difficile de trouver deux positions qui soient plus opposées que la sienne et celle de Descartes sur la question de savoir ce qui confere aux mathématiques la sûreté et la facilité particulières qu'elles semblent posséder et sur te que signifie le fail de cliercher à imiter le modèle des malhématiques dans les autres sciences et en particulier, puisque c'est bien le but qu'ils poursuivent l'un et l'autre, dans la philosophit elle-même. J'ai rappelé il y a un instant ce qu'était sur ce point la position de Descartes. Pour lui, les mathématiques s'occupent d'objets à propos desquels, à condition de prèter sulfisamment d'attention aux closes, qui peuvent être tenues à chaque fois entièrement sous le regard de l'esprit, et de procéder avec méthode en allant toujours du plus simple au plus complexe, nous sommes pratiquement dans l'inpossibilité de nous tromper. Leibniz pense que c'est, au contraire, parce quelles ont réussi beaucoup plus tôt que les autres sciences à rendre sensibles et, du même coup, mécaniques les raisonnements abstraits qu'elles utilisent, autrement dit à se dispenser de l'attention portée aux choses elles-mémes, pour la remplacer par des manipulations effectuées sur les signes, que les mathématiques ont pris une avance si considérable.

Selon lui, comme le dit Hacking, la véritable nature de la démonstration ne pouvait pas être comprise, tant que la géométrie était restée le paradigme de la rigueur mathématique. Car les démonstrations géométriques, qui impliquent le recours à des figures, ont une validité qui donne l'impression de dépendre de façon essentielle de leur contenu. Or Descartes a réussi à algébriser la géométrie, et l'algébrisation implique la possibilité d’éliminer un certain contenu, qui semblait essentiel. Grâce à la découverte de Descartes, la démonstration géométrique pouvait être traitée dorénavant, elle aussi, comme purement formelle. Mais Descartes s'est arrêté en chemin et n'est pas allé jusqu'à l'idée d'une Caractéristique Universelle Abstraite qui servirait de mode dexpression aux démonstrations dans tous les domaines et permettrait de les tester commodement et infailliblement. On pourrait évidemment ètre tenté de considérer l'idée de la mébhode elle-même comme un premier pas qui a été elfectui dans ce sens. Et c'est plus ou moins de cette façon que l'interprète Valéry. "Une méllode, écrit-il, n’est pas une doctrine: elle est un systeme d'opérations qui fasse mieux que l'esprit lui-mème le travail de l'esprit. Ce sont done nécessairement des opérations quasi matérielles, c'est-ì-dire que l"on peut concevoir, sinon rüaliser, au moyen d'un micanisme. Une doctrine peut pretendre nous enseigner des choses dont nous ne savions absolument rien; cependant qu une méthode ne se halle que d'opírer des transiormations sur ce dont nous savons dejà quelque partie pour en extraire ou en composer tout ce que nous pouvons

${ }^{10}$ Heinrich Scholz "Leibniz" (1942), in M/khesis Uninersalis, Abland!ungen zur Philosophie als strenge Wissenschaft, zweite Autlage, Wissemsclastliclat Butchgesellschaft, Darmstadt, 1969, p. 142. 
en savoir." "l Mais, pour Descartes, si la recherche de la vérité devient à la fois plus facile et plus sûre en devenant plus méthodique, ce n'est pas parce que la méthode remplace d'une façon quelconque le travail de l'esprit, mais, au contraire, parce qu'elle lui permet d'être totalement présent à ce qu'il fait et de rester entièrement maitre de ce qui se fait.

Leibniz a une tout autre idée de ce que nous pouvons attendre de la vraie méthode. Dans la lettre à Oldenburg du 28 décembre 1675, il écrit:

\begin{abstract}
Nous avons l'impression de penser (confusé|nert, s'entend) bien des choses qui pourtant impliquent contradiction: par exemple le nombre de tous les nombres. Nous devons avoir bien des soupcons a l'égard de la notion de l'infini, et du plus petit, et du plus grand, et du plus partait, et du tout (ommitas) lui-mème. Et il ne faut pas se fier à ces notions avant qu'elles ne soient soumises à ce critere qu'il me semble reconnaitre et qui cornme par un procédé mécanique rend la vérité fixe et visible (et pour ainsi dire irrésistible), lequel par un bienfait inexplicable nous a été octroyé par la nature.

Cette algèbre, dont nous faisons à bon droit un si grand cas, n'est qu'une partic de cet art gènéral. Elle nous foumit nėanmoins ceci que nous ne pouvons mime pas nous tromper si nous le voulons. Et que la vérité est attrapée comme quasiment peinte, exprimée comme à l'aide d'une machine sur le papier. Mtais je reconnais, quant à moi, que tout ce que l'algèbre démontre dans son genre n'est que le bánéfice dû à une science supérieure; une science que j'ai maintenant l'habitude d'appeler Caractèristique Combinatoire."12
\end{abstract}

On a l'habitude de considerer que l'innovation principale qu'apporte la révolution cartésienne par rapport à la tradition scolastique consiste dans la decision de faire de la certitude mathématique le modile de toute certitude et de la méthode que les mathématiciens utilisent pour y parvenir la méthode qui doit ètre suivie dans tous les cas. Marion écrit à ce sujet: "Au licu de la dialectique, que disqualifient ses conclusions seulement probables, il faut acquérir "une connaissance et une certitude égales à celles que peuvent produire les règles de l'Arithmétique", c'est-à-dire "introduire la certitude et l'évidence des démonstrations mathématiques dans les matières de Philosophie": I'universalisation du type d'évidenee et de certitude que montrent les mathématiques - universalisation qui relève, pour cela mème, d'une instance méta-mathénatique - constitue le seul véritable dépassement de l'Ecole (puisqu'il en récuse le mode de raisonnement, et non plus seulement telle ou telle conclusion), voilà l'apport proprement novateur de Descartes" (op. cit., p. I55).

Or Leibniz a unc vision de la situation et du rôle historique de Descartes qui est sensiblement diffërente de celle-là. Il pense que, si Descartes avait récllement pour ambition de généraliser à toutes les autres sciences et à la philosophie elle-mëme les avantages de la métlode mathématique, il a commis l'erreur majeure d'ignorer ce que ses prédécesseurs aristotéliciens et scolastiques avaient déjà réalisé dans ce sens-là et a été victime sur ce point du préjugé qui consiste à opposer le verbalisme et la stérilité de la logique formelle à la fécondité des mathématiques. Dans la lettre autobiographique à Gabriel Wagner, qui date de la fin de l'aunéc 1696, après avoir constaté que les présentations usuelles de la logique traitent successivement du concept, du jugement et raisonnentent, il observe que cette dernière partic, la theoric de l'inférence, $n$ 'a généralement pas bonne presse. On la considere habituellement comme triviale, ennuyeuse et stérile, parce qu"on continue à se la représenter essenticllement sur le modele de la théorie syllogistique. C'est une erreur regrettable, parce que, si les métlodes de logique per-

11 Paul Valśn, "Une vue da Descantes", in CEnres, 1, édition établic et annotée par Jean Hylier, Bibliotlieque de la Pléiade, Gallimard, Paris, 1957, p. 821.

12 Mcothematische Schrifien, lierausgegeben von C. J. Gerhardt, Georg Olms, Hildesleim-New York, 1971, I, p. 85.86 . 
fectionnées qui sont déjà à l'auvre dans les sciences récllement démonstratives ćtaient complètement expliqukes, clles feraient apparaitre la technique de l'inférence syllogistique comme śtant à peu près du mème niveau que le calcul de très petits nombres, que l'on fait sur ses doigts ou avec des bâtons. "Dans toutes les sciences infaillibles, ccrit Leibniz, lorsqu'elles sont dímontries exactement, sont pour ainsi dire incorporées des formes logiques supírieures, qui pour une part découlent des formes aristotéliciennes, pour une autre recourent en plus à autre chose" ( $P / h i l . S c / r .$, VII, p. 519). Il n'en est pas moins vrai que les règles du syllogisme sont les règles élémentaires que l'on doit imperativement connaitre avant de passer à des règles d'inférence plus compliquíes. D'Aristote, qui a eu le mérite éminent de soumettre les formes syllogistiques à un petit nombre de lois infaillibles, Leibniz dit, d'une façon qui a de quoi surprendre un lecteur lrabitué à voir les choses à la façon de Descartes et de ses héritiers modernes, qu'il a été, de ce fait, "le premier qui ait écrit mathématiquement en dehors des mathémasiques" (ibid.). Ecrire mathimatiquement en deliors des mathsmatiques voulait dire, justement. écrire, sur des sujets qui ne sont pas mathématiques et peuvent même être quelconques, sous forme d'argumenta in forma. Ce qu'a fait Aristote avec la thiorie du syllogisme n'ítait évidemment qu'un début. Mais Descartes a eu tort de croire, et de croire précisément au nom de l'ideal mathématique dont il se réclamait et qu"il cherchait à imposer à toutes les autres sciences que ce dibut devait être considéré en mỉme temps comme une fin et que le chemin qu'il convenait de suivre désormais était à pet près le contraire de celui-lit.

Je n'ai mallreureusement pas le temps de discuter ici récllement le probleme dilficile que pose l'impression, que pourrait donner la doctrine cartésienne de la críation des vérités éternelles, que les vérités nécessaires ont été rendues nécessairement vraies par une décision qui $n$ 'a pas de rapport essentiel avec le tait qu'elles soient démontrables, mėme si, dans les faits, nous ne pouvons géníalement les reconnaitre comme nécessairement vaies que par la dímonstration. Je me contenterai done simplement de remarquer qu'il est certainement très exagés de dire, comme le fait llacking, que, pour Descartes, la démonstration est sans pertinence pour la vérilé et plus encore que son utilité est uniquement celle d'un dispositif rhétorique ou psychologique conçu pour nous conduire à la perception de la vérité. Car. dans ce cas-lí, la vériț., une fois reconnue, devrait être en principe séparable du processus facultatif et éminemment contingent par lequel nous avons ctí amenés à la percevoir. Or mêne Descartes ne peut pas croire et ne croit manifestement pas que ce soit le cas. Ce qui est vrai est que la déinontrabilité n'est pas une condition nécessaire de la vérité non seulement paré que toute démonstration doit partir de propositions qui ne sont pas demontrables et doivent etre reconnues comme vraies d'une autre manière, mais également parce que ce n'est pas en tant que telle, mais seulement en tant cuelle est capable de nous procurer une perception elaire et distincte de la vérits qu'elle constitue une garantic de celle-ci. Et elte n'est pas non plus une condition sultisante, puisque, si nous n'étions pas certains de l'existence de Dieu et du filit qu’il ne peut pas itre trompeur, nous ne pourrions pas non plus itre certains que les propositions auxquelles nous sonumes conduits a donner notre assentiment sur la base de lit demonstration sont ellectivement vraies.

C’est ee ylut a conduit Descartes à aflïmer qu'en toute rigueur les païens ne pouvaient pas être certains de la vérité des assertions mathématiques qu ils formulent. Leibniz trouve cette doctrine absurde, parce que le principe de non-contradiction est le londement unique de toute la logique et que douter voudrait dire, en l'oceurrence, essiyer de penser contre le principe de non-contradiction, ee que Dieu lui-méme ne peut pas faire: "Car conme les expériences internes sont le fondement de toutes les vérites de fitit, ainsi le principe de contradiction 
est-il le fondement de toutes les vérités de raison; lui ôté, tout raisonnement est atteint, ct l'on ne peut plus rien conclure de Dieu ou d'aucune autre chose. C'est pourquoi rien n'est si absurde que d'assurer que l'on ne peut pas avoir la science certaine des vérités mathématiques sans une connaissance prialable de Dieu; si absurde que ceux qui savent les finesses de Descirtes le souponnent ici de je ne sais quel fâcheux artifice" (Phil. Schr., IV. p. 327). Pour Leibniz, de la contradiction à l'impossibilité (absolue), la conséquence est bonne. Elle ne l'est pas pour Descartes. Il ne suflit pas qu'une chose implique une contradiction dans notre pensce pour que nous soyons autorisés à la considérer comme impossible, même si, une fois que nous sommes assurés de l'existence de Dieu, nous n'avons plus de raison de conserver un doute quelconque sur ce point. Nous n'avons pas tort de considérer comme impossibles les choses qui impliquent contradiction. Elles le sont réellement. Ce qui est vrai est seulement qu'elles ne sont pas néccssairement impossibles. 